\title{
Experiences of termination of pregnancy in a stand-alone clinic situation
}

\author{
Caroline Boorer, MB BS, MFFP, Clinical Medical Officer, Central Nottinghamshire Healthcare NHS Trust and South \\ Lincolnshire Healthcare NHS Trust, UK \\ Judy Murty, MB ChB, DRCOG, MFFP, Senior Clinical Medical Officer, Leeds Family Planning Services, Leeds Community and \\ Mental Health Teaching Trust, Leeds, UK
}

Correspondence: Dr Judy Murty, 19 Swinsty Court, Clifton, York, YO30 5ZP. Tel: 01904 692518, Fax: 01904 692518, email: murty@easynet.co.uk

(Accepted September $12^{\text {th }}, 2000$ )

\begin{abstract}
Summary
This paper describes the authors' experience of conducting termination of pregnancy on conscious patients in community settings. If patients are appropriately selected and prepared, and the procedure conducted in the presence of well-trained and motivated nursing assistance, the method described is successful, safe and acceptable to patients.
\end{abstract}

Key words

community setting, local anaesthetic, termination of pregnancy

\footnotetext{
Key message points

- Local anaesthetic procedures provide an acceptable service for women requesting a termination of pregnancy.

- Local anaesthetic procedures can be provided in community settings.

- The procedure is safe and effective.
}

\section{Introduction}

Termination of pregnancy (TOP) can be carried out successfully on conscious patients in a stand-alone clinic situation, providing strict criteria are decided upon and adhered to. This is a review of our own personal experiences when operating in stand-alone clinics in a community setting. Previous experience has shown that clinicians from a family planning background can competently provide a TOP service. ${ }^{1}$ The experience described takes the procedure one stage further, into a community setting.

\section{History of procedure}

Various procedures have been developed for TOP, including vacuum aspiration under general and local anaesthetic. Even though it is common practice to provide a local anaesthetic procedure elsewhere, in the UK this is not routine.

Initially the procedure was introduced in the UK in the 1970's after use in the USA. ${ }^{2-5}$ Due to the difficulties of detecting pregnancy early enough, the procedure was equivalent to menstrual regulation. Doubt was cast on the legality of the procedure at this time, and further development in this country did not take place. However, development went ahead in other countries, including the USA and Holland.

\section{The procedure}

The method we have used is manual vacuum aspiration using an IPAS syringe and a flexible Karman type canulae.
Minimal local anaesthesia was provided, if needed, using $1 \%$ lignocaine gel. Recovery after the procedure was generally quite rapid; usually the patient was ready to leave within half-an-hour of the procedure and felt fit and able to carry out normal activities of life.

When performing abortions in a stand-alone clinic setting, it is vital that the choice of patients for this procedure is made following clear and unambiguous criteria, as follows. Firstly, it is most important that the patient is told clearly and honestly what to expect. This is not a pain-free procedure, but the well-prepared patient can cope well. Secondly, we used ultrasound to date each pregnancy and ascertain whether it was intra-uterine, especially with the very early gestations. If there was any doubt as to where the pregnancy was, further diagnostic ultrasound examination was advised. The optimum time to perform this procedure, in our opinion, is between 6 and 10 weeks gestation. Prior to 6 weeks the possibility of missing the pregnancy is higher, and the procedure can be more painful, and after 10 weeks it tends to take longer. The chance of complication, such as potential for increased postoperative bleeding or risk of perforation, is higher with later gestations. 6

Well-trained and motivated nursing assistance is of great importance to the success of running such a service. Prior to the procedure such nurses can explain all aspects to the patient, during it they can provide 'vocal local' to help and reassure, and afterwards they can monitor the patient and ascertain readiness for discharge. This requires a calibre of nurse akin to the best family planning trained nurse, who is an able assistant and support when one is fitting an IUD.

Because the woman is awake and fully aware of having the TOP, it can be emotionally challenging for the staff, as this is the time that a woman can express her hidden feelings about the procedure.

We have performed an average of 800 procedures per year each over the past 3 years. Our results show a continuing pregnancy rate of less than $1 \%$ (principally in the very early gestations) and a re-operation rate, after disruption of the pregnancy, of around 2\% (principally in the later gestations). This compares with the best figures of 2.3/1000 for surgical TOP under general anaesthetic and 6/1000 using Mifegyne. ${ }^{6}$ With experience those procedures performed between 6 and 10 weeks were nearing $100 \%$ success rate. Also, we did not experience any perforations.

Although abortion is always likely to be an emotive and difficult decision for most patients, it does not need to be a lengthy and debilitating procedure when performed in early pregnancy. We feel that the method described is a very 
successful, safe and acceptable procedure for those patients who choose to be awake, and fit the criteria outlined. It is ideally suited to community clinics, provided that there is gynaecological back-up immediately available in (the rare) case of need.

\section{Statement on funding and competing interests}

Funding. None declared.

Competing interests. Both authors have worked for Marie Stopes International. The article is purely a reflection on the authors own personal experiences and do not represent the views of any commercial or charitable organisation.

References

Randall S. Provision of a district abortion service by a community family planning department. British Journal of Family Planning 1996; 21: 152 .

Brenner WE, Edelman DA, Kessel E. Menstrual regulation in the United States: a preliminary report. The Journal of Family Planning Doctors 1975; 1 (2): Iand 8 .

Stringer J, Anderson M, Beard RW, et al. Very early termination of pregnancy (Menstrua

Lewis SC, Lal S, Branch B, et al. Outpatient termination of pregnancy. British Medical Journal McGarry J Mininal

作 